\title{
Erratum: Delta-Function Expansion of Mayer Function with Application to Virial Coefficients ${ }^{1}$
}

\author{
James C. Rainwater ${ }^{2}$
}

Received February 28, 1981

In the enumeration of the nonzero coefficients $C_{i j k}$ [Eq. (26)], $C_{330}=-4$ was overlooked. Inclusion of this coefficient requires the addition of a term $(8 / 9) R_{3}^{2}$ inside the bracket of Eq. (32). When modified, the expression for $C$ is consistent with Kihara's solution ${ }^{(1)}$ for the square-well third virial coefficient when the diameter ratio is near unity. Equations (38) and (39), when modified accordingly, agree totally with Bruch's upper bound ${ }^{(2)}$ on $C$ for the exponential potential, as they should from an analysis of the ordering of the expansion.

The missing term is sixth order in softness and thus numerically very small, so the qualitative conclusions drawn in the paper, and a subsequent discussion, ${ }^{(3)}$ remain valid.

The proof of Appendix A is not correct since, as written, the set $\left\{l_{i}\right\}$ must contain $j$ elements and thus the sum over such sets cannot be taken outside the $j$ sum. This defect may be remedied by grouping together those sets which are identical upon removal of their zero elements and then reducing the sum for each new grouping to the form of Eq. (A5). Alternately, the result follows immediately from a Taylor series expansion in $x$ of the identity ${ }^{(4)}$

$$
R_{m}=\sigma^{m}(\epsilon / k T)^{m / n} \int_{0}^{\infty} d z e^{-z}\left[z^{-x}-\Gamma(1-x)\right]^{m}
$$

\footnotetext{
${ }^{1}$ This paper appeared in J. Stat. Phys. 19(2), 177 (1978).

${ }^{2}$ Thermophysical Properties Division, National Engineering Laboratory, National Bureau of Standards, Boulder, Colorado 80303.
} 


\section{REFERENCES}

1. T. Kihara, Rev. Mod. Phys. 25:831 (1953).

2. L. W. Bruch, Phys. Fluids 11:1938 (1968).

3. J. C. Rainwater, J. Chem. Phys. 71:5171 (1979).

4. R. F. Kayser, private communication. 